\title{
Correlación geoquímica entre crudos y rocas del sistema petrolero de la península de Santa Elena y el golfo de Guayaquil
}

\author{
Erica Lorenzo $^{1 *}$; Wladimir Andrés Roca-Beltrán ${ }^{1}$; Manuel Martínez ${ }^{1}$; Antonio Morato1; \\ Paulo César Escandón-Panchana ${ }^{1}$; Cristian Paul Álvarez-Domínguez ${ }^{1}$
}

DOI: http://dx.doi.org/10.18273/revbol.v40n1-2018002 @ (c)

Forma de citar: Lorenzo, E., Roca-Beltrán, W.A., Martínez, M., Morato, A., Escandón-Panchana, P.C., y Álvarez-Domínguez, C.P. (2018). Correlación geoquímica entre crudos y rocas del sistema petrolero de la península de Santa Elena y el golfo de Guayaquil. Boletín de Geología, 40(1), 31-42. Doi: 10.18273/revbol.v40n1-2018002.

\section{RESUMEN}

El área suroeste de la costa ecuatoriana es una región tradicionalmente petrolera, sin embargo aún no se ha definido cuál es el sistema petrolero reinante en la región. Entre las principales rocas propuestas como generadoras de los crudos del litoral, se encuentran las formaciones Seca y Socorro del Eoceno así como Dos Bocas y Subibaja del Mioceno. Con la finalidad de aportar nuevos elementos al conocimiento de la zona, fueron estudiados tanto crudos como rocas provenientes de campos onshore y offshore, usando técnicas geoquímicas que incluyen cromatografía de gases e isótopos de carbono. Parámetros como el Índice Preferencial de Carbonos (IPC), Pri/ $n-\mathrm{C}_{17}, \mathrm{Fit} / n-\mathrm{C}_{18} \mathrm{y}$ las distribuciones isotópicas, indican que los crudos de los campos onshore están genéticamente diferenciados de los crudos offshore, y se asocian con una roca madre clástica con aporte de materia orgánica mixta, depositada en ambiente subóxico, consistente con la parte distal de un complejo estuarino. Por otro lado, las rocas analizadas son inmaduras térmicamente y con muy bajo potencial generador, por lo que no pueden ser correlacionadas con los crudos analizados, tal y como se venía haciendo tradicionalmente.

Palabras clave: geoquímica; roca madre; madurez térmica; Golfo de Guayaquil; Ecuador.

\section{Geochemical correlation between oils and rocks of the petroleum system of the Santa Elena peninsula and the gulf of Guayaquil}

\begin{abstract}
The southeastern of the Ecuadorian coast is a traditionally oil producing region, but the petroleum system has not been defined yet. The proposed main source rocks are Seca and Socorro formations (Eocene) and Dos Bocas and Subibaja formations (Miocene). In order to contribute new elements to the knowledge of the area, both crude and rock from onshore and offshore fields were studied, using geochemical techniques that include gas chromatography and carbon isotopes. Parameters such as the Carbon Preference Index (IPC), Pri $/ n-\mathrm{C}_{17}$ and $\mathrm{Phy} / n-\mathrm{C}_{18}$ ratios and the isotopic distributions, indicate that onshore oils are genetically differentiated from offshore oils and are associated with a detritic source rock with mixed organic matter, deposited in a suboxic environment, consistent with the distal area of an estuarine complex. On the other hand, the rocks analyzed are thermally immature and with very low generating potential, so that they cannot be correlated with the crude analyzed, as was traditionally done.
\end{abstract}

Keywords: geochemistry; source rock; thermal maturity; Gulf of Guayaquil; Ecuador.

\footnotetext{
${ }^{1}$ Universidad Estatal Península de Santa Elena (UPSE), La Libertad, Ecuador. (*)elorenzo@upse.edu.ec; wladimir_andres_17@gmail.com; manmarti@gmail.com; amorato@upse.edu.ec; pcescandonp@hotmail.com; calvarez@upse.ēu.ec
} 


\section{INTRODUCCIÓN}

El área del Golfo de Guayaquil se ubica en el suroeste de la costa ecuatoriana y cubre aproximadamente 15.000 $\mathrm{km}^{2}$. La roca madre de petróleo es desconocida, aunque han sido propuestas muchas unidades para cumplir dicha función (Benítez, 1995; Jaillard et al., 1995; Deniaud et al., 1999). Las principales acumulaciones petroleras se encuentran en rocas de edad Eocena (Grupo Azúcar) y Miocenas (Formaciones Seca y Socorro). Por otro lado, la región conocida como "Campo Amistad" se localiza próxima a la isla de Puná entre la Cuenca Jambelí y el Alto de Santa Elena al suroeste del golfo, a unos $60 \mathrm{~km}$ al oeste de Machala (Benítez, 1995) (FIGURA 1).
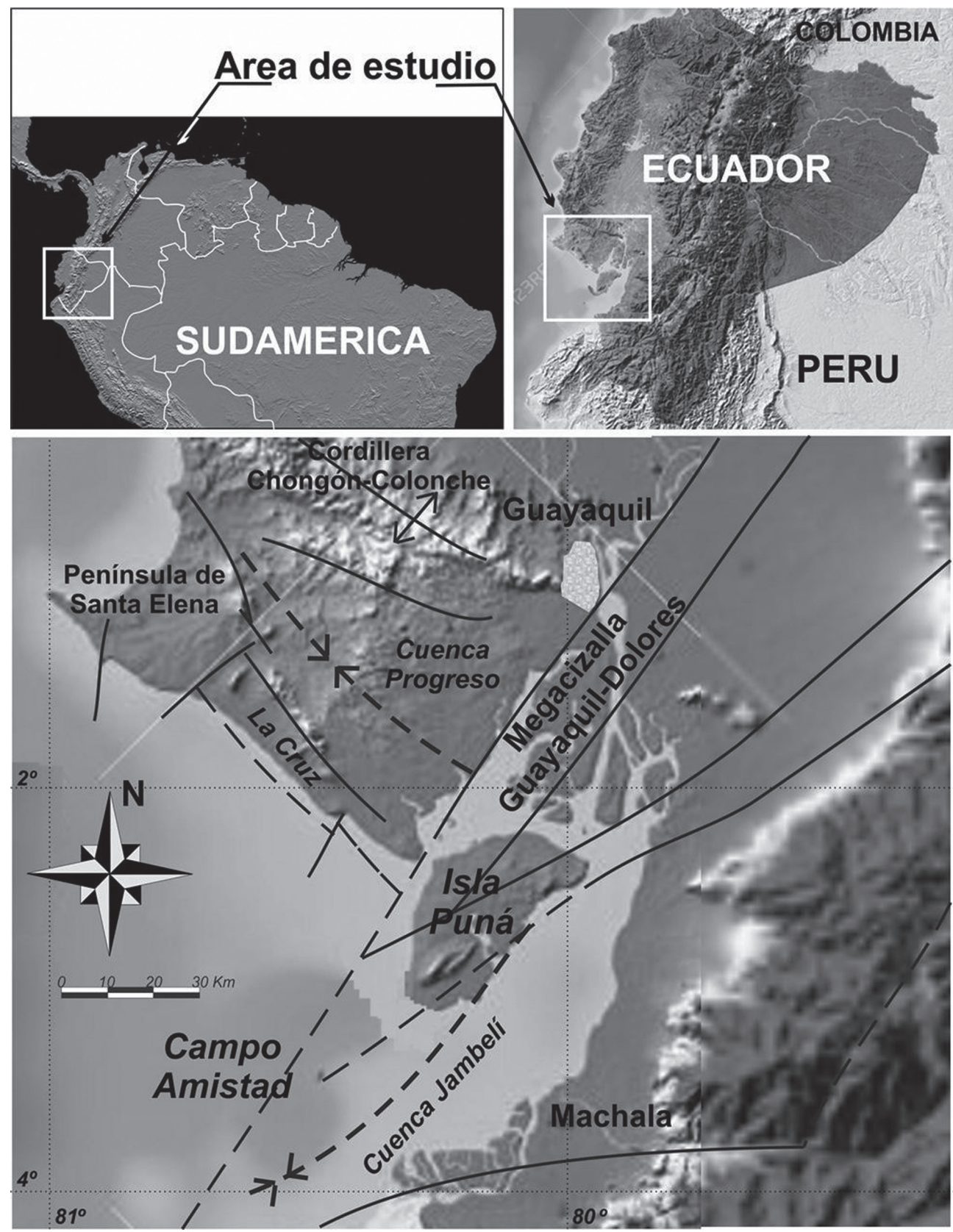

FIGURA 1. Ubicación de la zona objeto de estudio.

La Península de Santa Elena ha sido dividida en varias regiones; la más importante de ellas desde el punto de vista petrolífero es el bloque Gustavo Galindo Velasco, con un área de $70 \mathrm{~km}^{2}$. Este bloque está dividido en tres zonas, norte, central y sur, y algunos de sus campos más importantes son: Ancón, Petrópolis y Santa Paula. 
Estos campos poseen crudos de importancia económica para el país, y entre ellos destaca el Campo Ancón, cuyos crudos presentan una gravedad API de $35^{\circ}$ en promedio, lo que los clasifica como crudos livianos (Cobos, 2010). Sin embargo, se conoce muy poco de sus características geoquímicas, las cuales pueden contribuir a los estudios de exploración, con miras a futuras explotaciones en este campo.

Este trabajo presenta el estudio geoquímico de cinco muestras de crudos, cuatro de ellos procedentes del área de la Península de Santa Elena (onshore) y una del Golfo de Guayaquil (offshore). Los datos de esta última se han usado como referencia a la hora de extrapolar los datos y comparar si todas pertenecen a un mismo sistema petrolero. Así mismo fueron analizadas 20 muestras de rocas procedentes de 5 pozos, ubicados offshore y onshore (FIGURA 1). La finalidad de este trabajo es obtener información sobre el origen de la materia orgánica precursora de los crudos y del tipo de roca fuente que los originó, del grado de madurez alcanzado por la roca fuente y los posibles procesos de alteración que ocurrieron en estos crudos en el yacimiento.

\section{MARCO GEOLÓGICO}

La geología del suroeste de Ecuador ésta condicionada por la interacción de las Placas Nazca y Sudamericana (Jaillard et al., 2009). En contexto general, muchos autores (Benítez, 1995; Deniaud et al., 1999; Cobos, 2010), han reportado la columna sedimentaria de la Península de Santa Elena y el Golfo de Guayaquil como unidades tectonoestratigráficas sobre un basamento oceánico de edad cretácica (Formación Piñón) (FIGURA 2).

\section{Cretácico Superior}

Cenomaniense-Coniaciense/Santoniense-Campaniense: Sobre la Formación Piñón se localiza un conjunto de rocas vulcanoclásticas y lutitas tobáceas que conforman la Formación Cayo, que a su vez puede ser subdividida en los miembros: Calentura, Cayo y Guayaquil. El miembro Calentura aparece sobre la Formación Piñón, como una gruesa sucesión de lutitas, limolitas negras laminadas y areniscas turbiditicas compuestas de clastos volcánicos y depositadas en ambientes pelágicos. En el área de Guayaquil, el miembro Cayo se depositó en disposición concordante sobre la Formación Calentura (Benítez, 1995; Jaillard et al., 2009). Las rocas que caracterizan a este miembro son grauvacas con intercalaciones de lutitas características de ambientes turbidíticos de alta densidad con sedimentos vulcanoclásticos. Finalmente, culminando la Formación Cayo se encuentra el miembro Guayaquil cuya composición principal está basada en tobas, arcillas y cherts negros, característicos de ambientes pelágicos con actividad volcánica distal (Marksteiner y Alemán, 1991; Wallrabe-Adams, 1990).

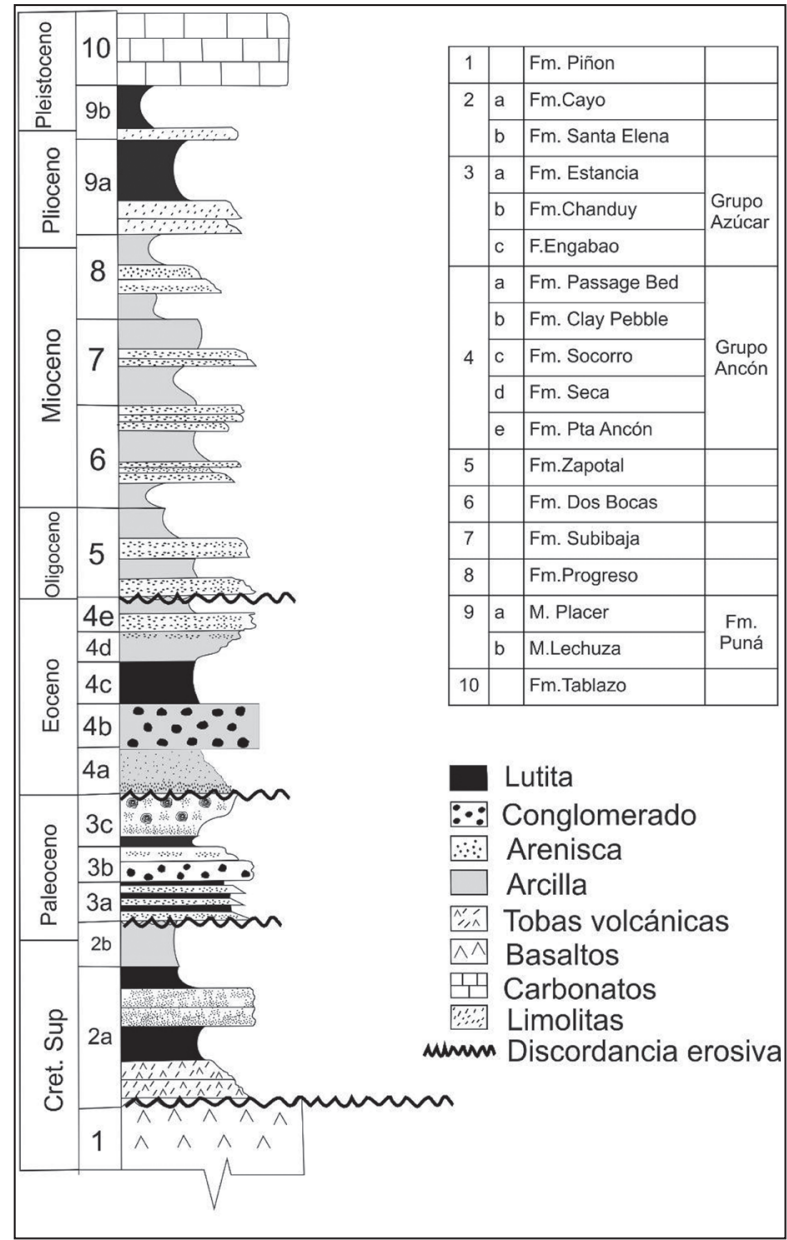

FIGURA 2. Columna Estratigráfica Progreso/Santa Elena integradas.

\section{Maastrichtiense-Paleoceno Temprano}

La Formación Santa Elena solo aflora en la península homónima. Ha sido descrita como una sucesión de depósitos turbidíticos finos, depósitos de deslizamientos por gravedad y flujos densos. Esta unidad es considerada con un equivalente al miembro Guayaquil (Jaillard et al., 1995; Deniaud, 1998).

\section{Paleógeno}

Para el Paleoceno Tardío-Eoceno Inferior, el Grupo Azúcar (FIGURA 2) ha sido reportado como una facies conglomerática que separa dos facies arenoarcillosa, características de ambientes de abanico submarino. Con base en la composición del grupo, 
este ha sido a su vez subdividido en 3 miembros: Estancia, Chanduy y Engabao. Cabe destacar por su importancia en producción petrolera el miembro Chanduy también denominado Formación Atlanta, que constituye el principal reservorio de la región (Benítez, 1995).

La sedimentación eocena en la Península de Santa Elena está representada por el Grupo Ancón, el cual se compone de tres formaciones; Formación Passage Beds, la Formación Clay Pebble Beds, la Formación Socorro, la Formación Seca y la Formación Punta Ancón. La Formación Passage Beds está compuesta por intercalaciones de arenisca calcárea de grano grueso y areniscas que se dispone discordante sobre el Grupo Azúcar.

La Formación Clay Pebble Beds corresponde a sedimentos turbíditicos característicos de corrientes submarinas de alta densidad. Esta formación incluye un banco de areniscas aislado denominado Formación Santo Tomas, el cual está considerado como nivel productor en el campo Ancón.

La Formación Socorro está constituida por lutitas oscuras y areniscas de carácter turbíditico, características de zona de transición entre abanicos de talud y canales de plataforma continental.

La Formación Seca depositada concordantemente sobre la anterior (FIGURA 2), está constituida por arcillas grises a verdosas características de depósitos de plataforma y abanicos submarinos. Por último, a este grupo pertenecen las areniscas con base erosiva de la Formación Punta Ancón que corresponden a depósitos marinos próximos al talud.

Tras las formaciones eocenas hay una discordancia erosiva que indica la pérdida de las formaciones de edad más joven, hasta llegar al Pleistoceno con la Formación Tablazo, la cual son niveles calcáreos marinos aterrazados. Dado que el estudio que se presenta abarca muestras en el Golfo de Guayaquil y la geología de la zona no ha sido reportada en columna estratigráfica, se ha completado la columna propuesta con los sedimentos de la Cuenca Progreso equivalente a las edades perdidas en la Península de Santa Elena. Estos son:

Oligoceno: En el Oligoceno se depositó una serie terrígena compuesta por areniscas, conglomerados, limolitas y arcillas, con alta variabilidad lateral y pobremente clasificadas que constituyen la Formación Zapotal.

Mioceno: El Mioceno inferior está representado por dos formaciones compuestas por arcillolitas grises correspondiente a un máximo transgresivo en el área Dos Bocas y Villingota. Los depósitos correspondientes al Mioceno medio son interestratificaciones de arcillolitas oscuras y areniscas gruesas mal seleccionadas. Por último, en el Mioceno superior se depositó la Formación Progreso, que se compone de interestratificaciones de areniscas y lutitas marrones, calcáreas y ricas en fósiles características de medios marinos poco profundos o de transición.

Plioceno: Esta edad está representada por el Miembro Placer de la Formación Puná, compuesto por intercalaciones de lutitas con limolitas y areniscas depositadas en ambiente estuarino.

Cuaternario: Sobre la unidad Pliocena, concordantemente se dispone el miembro Lechuza, cuya litología es similar al anterior salvo que al norte se intercalan con calizas dolomíticas depositadas en ambiente marino de plataforma somera.

\section{MUESTRAS Y MÉTODOS}

\section{Muestras}

Las muestras de crudos y rocas empleadas en el presente estudio, fueron colectadas en su momento por la hoy extinta Corporación Estatal Petrolera Ecuatoriana (CEPE) y almacenadas en el Laboratorio de Petróleo de la Universidad Estatal Península de Santa Elena. Las muestras fueron tomadas de los pozos cuya nomenclatura y ubicación aparecen en la FIGURA 3.

\section{Obtención de las fracciones orgánicas}

Los crudos fueron tratados con benceno en una proporción 1:1 a fin de remover agua y sedimentos. En el caso de las rocas, éstas fueron cuidadosamente pulverizadas en un equipo Shatterbox, con un vial de carburo de tungsteno, por tiempos muy cortos a fin de impedir posibles alteraciones por calentamiento. Una vez pulverizadas hasta pasar el tamiz de 150 mesh, fueron sometidas a extracción exhaustiva Soxhlet con diclorometano como solvente (aproximadamente 2 días). El extracto obtenido fue rotaevaporado y el bitumen obtenido fue pesado, para luego ser llevado a fraccionamiento. 


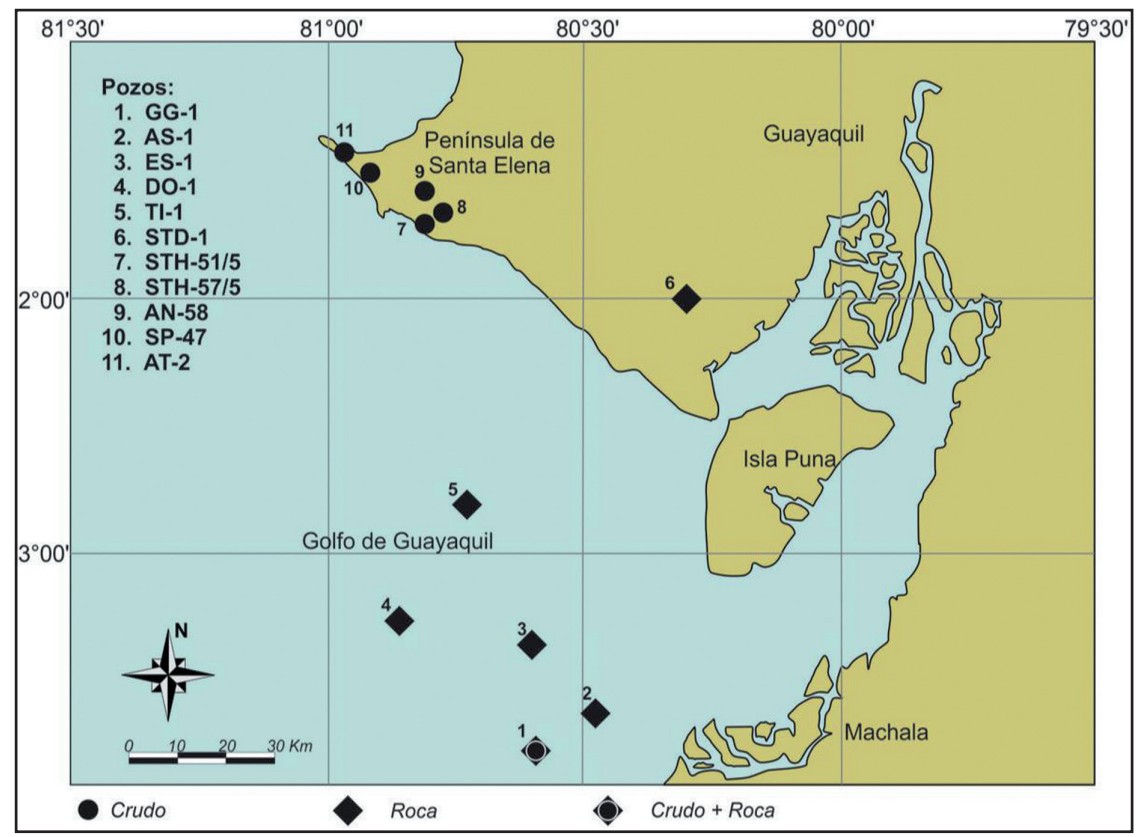

FIGURA 3. Localización de los pozos de estudio.

Una alícuota de cada muestra (crudo libre de agua y sedimentos o bitumen de roca) fue fraccionada en saturados, aromáticos, resinas y asfaltenos (SARA) mediante técnicas convencionales. Brevemente, los asfaltenos fueron separados con $n$-heptano en una relación 1:40 v/v y haciendo uso de un papel de filtro Whatman's No2, según la norma ASTM D3279-07. Los asfaltenos fueron purificados varias veces por extracción Soxhlet con $n$-heptano hasta obtener el líquido incoloro.

\section{Determinaciones analíticas}

El contenido de azufre fue llevado a cabo usando un analizador de azufre Leco SC-432. Los análisis cromatográficos fueron efectuados en un equipo Agilent $6890 \mathrm{~N}$ con una columna HP-1 de $30 \mathrm{~m}$ de longitud x 0,25 $\mathrm{mm}$ de diámetro interno, $\mathrm{y}$ espesor de la película interna de $0,25 \mu \mathrm{m}$. El horno fue programado de $35^{\circ} \mathrm{C}$ por 15 minutos; incrementado luego a $270^{\circ} \mathrm{C}$, a $30^{\circ} \mathrm{C} / \mathrm{min}$, luego a $300^{\circ} \mathrm{C}$ a $10^{\circ} \mathrm{C} / \mathrm{min}$, usando Helio como gas de arrastre. Los biomarcadores fueron identificados por comparación de los espectros de masa, tiempos de retención y por iones diagnósticos disponibles en la literatura especializada (Peters et al., 2005).

Las determinaciones isotópicas de carbono sobre las fracciones saturadas y aromáticas fueron llevadas a cabo en un equipo analizador elemental Thermo Finnigan 1112 acoplado a un espectrómetro de masas Finnigan Mat Delta C. Se emplearon como materiales de referencia el grafito USGS 24, sacarosa IAEA-CH6, polietileno IAEA-CH7 y crudo NBS-22. Los valores de la relación $\delta^{13} \mathrm{C} / \delta^{12} \mathrm{C}$ se reportan respecto al patrón PDB. Todos los análisis fueron llevados a cabo por duplicado.

\section{RESULTADOS Y DISCUSIÓN}

\section{Caracterización de los crudos}

Las características generales de la composición, el total de azufre y la gravedad API de las cinco muestras de crudo analizadas se muestran en las TABLA 1. Del análisis porcentual de las fracciones del crudo, se obtuvo datos similares para las 5 muestras analizadas: la fracción alifática de hidrocarburos (SAT) arrojó valores entre el $49,2 \%$ y el $69,1 \%$ (FIGURA 4 ); los hidrocarburos aromáticos (ARO) en el intervalo 4,4-20\%; mientras que la fracción polar (POL) presenta valores entre el 10,4 y el $18,3 \%$ (TABLA 1). Los crudos analizados, no muestran signos de alteraciones por biodegradación dado el alto porcentaje de saturados que presentan. Sin embargo, no se puede descartar rotundamente la ausencia de tal alteración secundaria ya que una eventual mezcla de crudos puede enmascarar el proceso de biodegradación (Escobar-Navarro, 2008). Por otra parte, la distribución de las muestras de crudo respecto a las relaciones Pri $/ n-\mathrm{C}_{17}$ y Fit $/ n-\mathrm{C}_{18}$ revela distintos grados de alteración para los crudos analizados, de tal forma que para el SP47 parece que el grado de biodegradación es mayor que para el crudo offshore GG-1 (FIGURA 5). Finalmente, la presencia del $\mathrm{C}_{29}$ 25-nor-17( $\alpha$ )-hopano, que deriva de la desmetilación del $\mathrm{C}_{30} 17(\alpha) 21(\beta)$-hopano (FIGURA 6) en el fragmentograma m/z 191 confirma la existencia de biodegradación en los crudos analizados. 
TABLA 1. Cuantificación en porcentaje de las fracciones esenciales y gravedad específica de los crudos analizados.

\begin{tabular}{ccccccc}
\hline Pozos & \% Saturados & \% Aromáticos & \% Resinas & \% Asfalteno & API & \%S \\
\hline SP-47 & 49,2 & 34,3 & 16,3 & 0,2 & 29,9 & 0,11 \\
AN-58 & 69,1 & 20,4 & 10,2 & 0,3 & 37,3 & 0,01 \\
STH-51 & 58,9 & 30,1 & 10,7 & 0,3 & 33,3 & 0,01 \\
STH-57 & 59,6 & 27,3 & 12,8 & 0,3 & 33,3 & 0,01 \\
GG-1 & 55,7 & 28,0 & 14,0 & 2,3 & 33,9 & 0,10 \\
\hline
\end{tabular}

Por otro lado, los valores obtenidos para la gravedad específica de los crudos bajo análisis se encuentran en el intervalo de $29,9-33,9^{\circ}$, lo que permiten inferir un nivel incipiente a intermedio en madurez térmica. No obstante, dado que se detecta alteración secundaria en el yacimiento, esta inferencia no es concluyente.

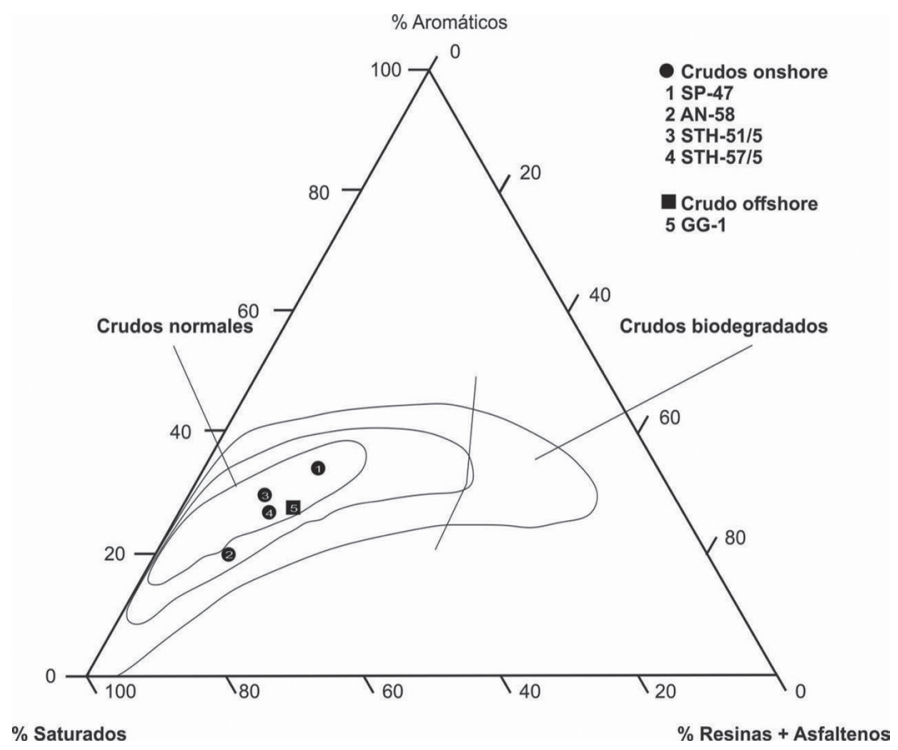

FIGURA 4. Diagrama triangular de composición S.A.R.A.

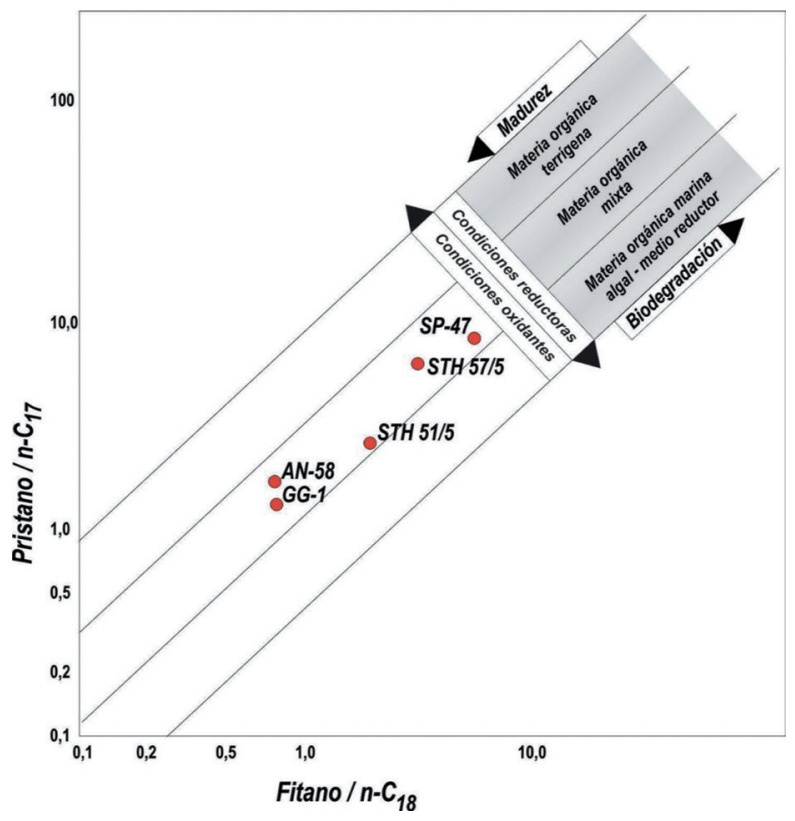

FIGURA 5. Diagrama de Pri $/ n-\mathrm{C}_{17} v s$. Fit $/ n-\mathrm{C}_{18}$ de los crudos estudiados.

\section{Distribución de $\boldsymbol{n}$-alcanos}

En las muestras estudiadas, la distribución de parafinas es variable, con predominio de $n$-alcanos livianos $(<n$ $\mathrm{C}_{20}$ ) sobre los pesados $\left(>n-\mathrm{C}_{20}\right.$, FIGURA 7).

El Índice Preferencial de Carbonos (IPC) pudo medirse en dos muestras, AN-58 y GG-1; los valores se encuentran en torno de la unidad $(1,07-1,2)$ indicando madurez moderada a alta para los crudos en estudio. Para el resto de crudos ubicados onshore, la ausencia de $n$-alcanos en el intervalo empleado por la ecuación del ICP impide efectuar el cálculo, lo que confirma la existencia de alteración secundaria de los crudos, posiblemente por biodegradación. En los crudos SP-47, STH-51/5 y STH$57 / 5$ queda evidente la ausencia de $n$-alcanos; sólo son detectadas las señales correspondientes a la familia de isoprenoides, desde el isoprenoide con 13 átomos de carbono, IP-13, hasta el fitano.

La distribución de $n$-alcanos, con predominio de los livianos sobre los pesados, apunta a materia orgánica 
marina; por otra parte, las relaciones Pri/Fit mayores a la unidad, pero en torno a 2 permiten proponer condiciones moderadamente reductoras a levemente oxidantes, propias de medios fluvio-marinos, con materia orgánica mixta. Este dato es coincidente con la información obtenida de la FIGURA 5, la cual agrupa todas las muestras de crudos obtenidas dentro del campo de materia orgánica mixta (Lijmbach, 1975; Hunt, 1996; Moustafa, 2004).

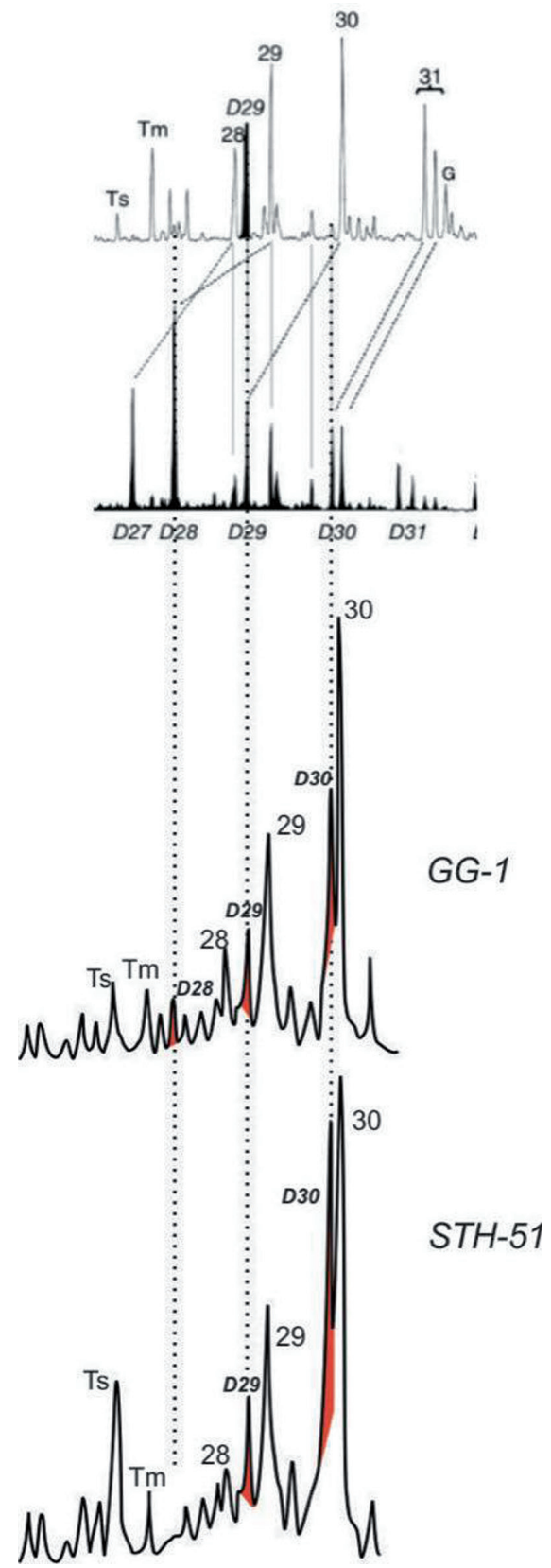

FIGURA 6. Comparativa de fragmentogramas $\mathrm{m} / \mathrm{z} 191$ correspondientes a los crudos STH-51 y GG-1. La imagen superior que sirve de referencia para la identificación proviene de una muestra de la Cuenca Oriental de Venezuela (Peters et al., 2005).
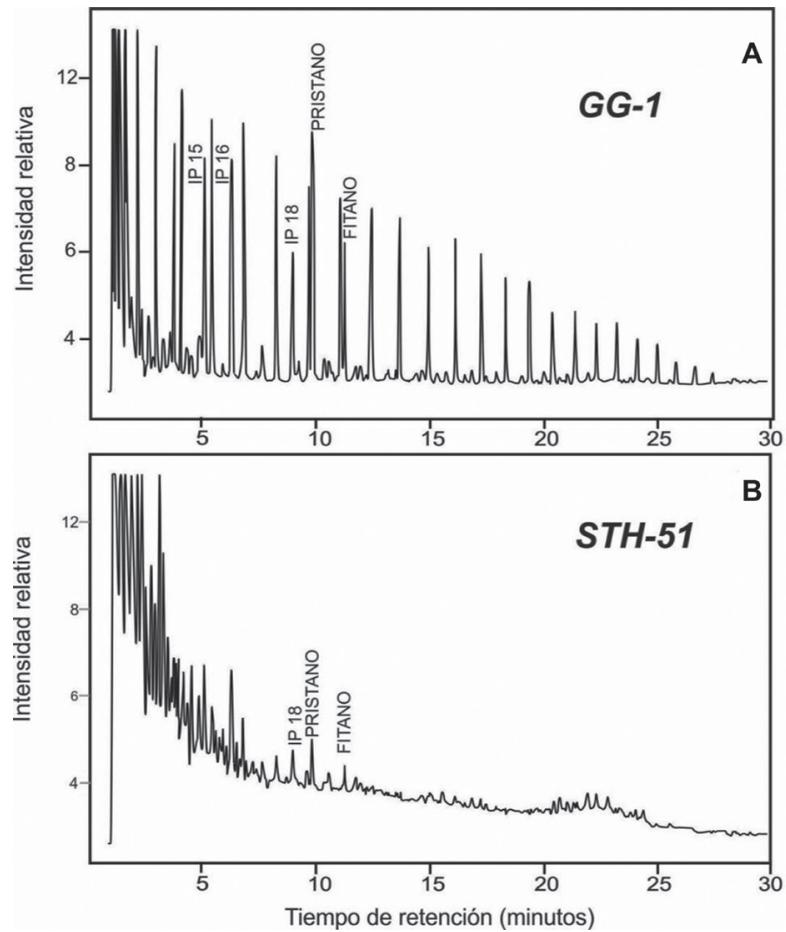

FIGURA 7. Cromatograma sobre la fracción saturada para las muestras: A. GG-1 y B. STH-51.

\section{Madurez térmica}

La madurez térmica de los crudos analizados fue establecida con base en: la relación $17 \alpha$-trisnorhopano/18 $\alpha$ - trisnorhopano (Ts/Tm), relación Moretano/Hopano $(\mathrm{Mo} / \mathrm{Ho})$, relación de los isómeros de bisnorhopano 33S/33R y el Índice preferencial de Carbono (IPC). Estos índices fueron calculados a partir de las áreas de las señales en los cromatogramas y fragmentogramas $\mathrm{m} / \mathrm{z} 191$ respectivamente (FIGURAS 7 y 8 ).

La relación $\mathrm{Mo} / \mathrm{Ho}$ reporta datos para todos los crudos, en el rango entre 0,12 y 0,18 , lo cual apunta hacia crudos maduros. El hecho de que los crudos analizados apunten tener cierta madurez está apoyado también por la relación Ts/Tm. Los valores obtenidos oscilan entre 1,04 y 2,5 , indicando que los crudos analizados cuentan con una madurez térmica mínima de entrada en la ventana de petróleo.

Por último, la relación de esteranos 22S/22R igualmente apunta hacia que los crudos analizados son maduros dado que los datos obtenidos se encuentran en el rango de 1,5 a 1,9 (Moustafa y Morsi, 2012) (FIGURA 9). 

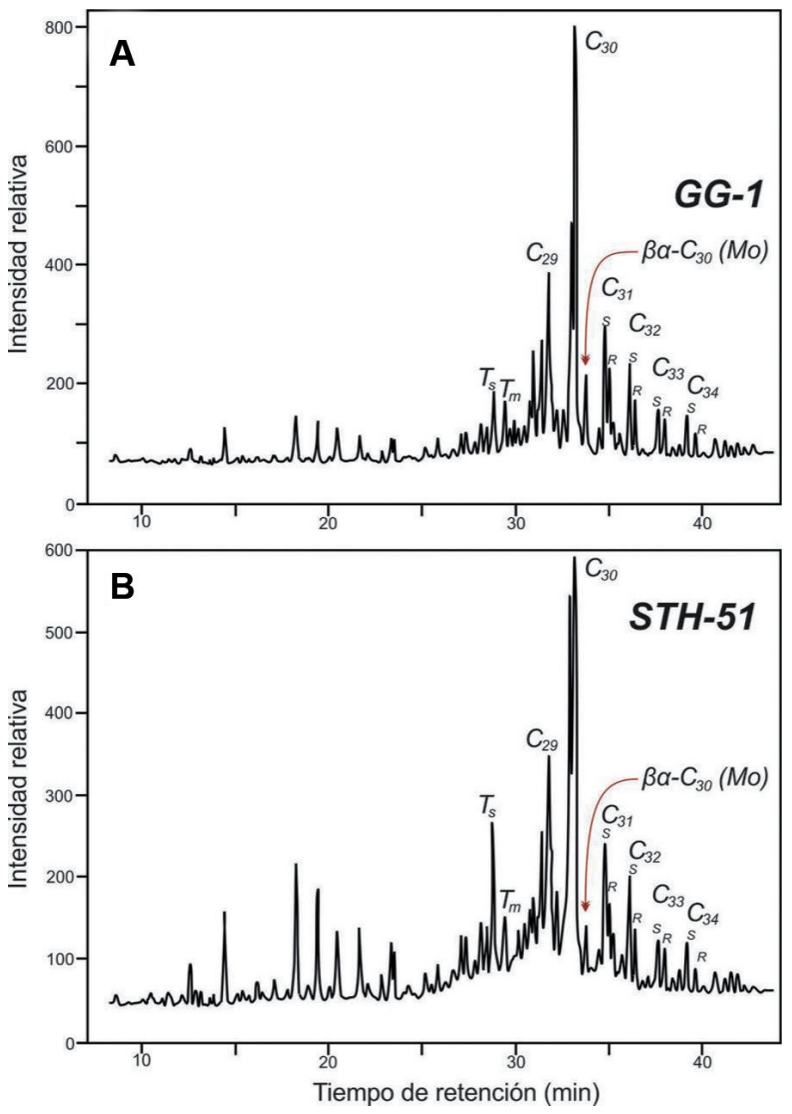

FIGURA 8. Fragmentogramas (GC-MS) $\mathrm{m} / \mathrm{z} \quad 191$ correspondientes a los crudos A. GG-1 y B. STH-51.

\section{Isótopos de carbono}

Los valores obtenidos del análisis de los isotopos estables del carbono, para los cinco crudos analizados se presentan en la TABLA 2. Los crudos SP-47 muestran un $\delta^{13} \mathrm{C}$ promedio de $-22,4 \%$ sin embargo el dato obtenido para el CG-1 es isotópicamente más liviano y se corresponde con un $\delta^{13} \mathrm{C}$ de $-25,3$. La representación gráfica de estos datos en el diagrama de Sofer (1984),
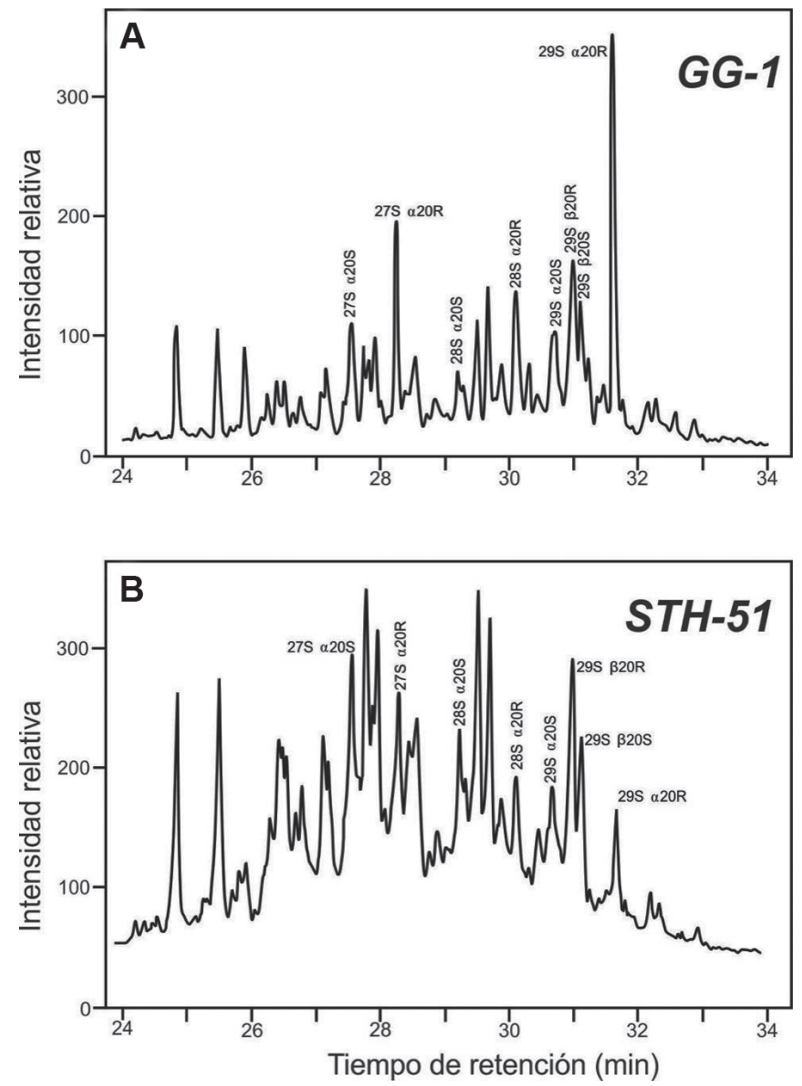

FIGURA 9. Fragmentogramas (GC-MS) $\mathrm{m} / \mathrm{z} \quad 217$ correspondientes a los crudos A. GG-1 y B. STH-51.

muestra la división de las muestras en dos familias isotópicamente similares, lo cual apunta hacia un mismo origen sedimentario de la materia orgánica que generó dichos crudos (FIGURA 10). Sin embargo, el hecho que el GG1 sea isotópicamente más liviano permite inferir un aporte significativo de materia orgánica terrestre, dato que se ve apoyado con la distribución de esteranos discutida en el siguiente apartado.

TABLA 2. Parámetros geoquímicos calculados a partir de los fragmentogramas y fraccionamiento isotópico de la fracción saturada y aromática.

\begin{tabular}{|c|c|c|c|c|c|c|c|c|c|c|c|c|}
\hline Pozos & IPC & Ts/Tm & Mo/Ho & $22 S / 22 R$ & Pri/Fit & $\operatorname{Pri} / n-C_{17}$ & Fit $/ n-C_{18}$ & $\% \mathrm{~S}$ & $\begin{array}{c}\delta^{13} \mathrm{C} \\
\text { Saturados }\end{array}$ & $\begin{array}{c}\delta^{13} \mathbf{C} \\
\text { Aromáticos }\end{array}$ & $\mathrm{CV}$ & API \\
\hline SP-47 & No disponible & 1,04 & 0,14 & 1,55 & 1,53 & 8,29 & 9,50 & 0,11 & $-22,3$ & $-21,7$ & $-3,41$ & 29,9 \\
\hline AN-58 & 1,07 & 2,17 & 0,135 & 1,83 & 1,94 & 1,41 & 0,81 & 0,01 & $-22,1$ & $-20,8$ & $-1,91$ & 37,3 \\
\hline STH-51 & No Disponible & 2,5 & 0,125 & 1,84 & 1,93 & 2,56 & 3,65 & 0,01 & $-21,6$ & $-21,5$ & $-4,73$ & 33,3 \\
\hline STH-57 & No Disponible & 2,5 & 0,13 & 1,9 & 1,60 & 6,91 & 4,76 & 0,01 & $-21,6$ & $-21,2$ & $-4,07$ & 33,3 \\
\hline GG-1 & 1,2 & 1,21 & 0,183 & 1,65 & 1,79 & 1,28 & 0,75 & 0,10 & $-25,3$ & $-24,0$ & $-0,92$ & 33,9 \\
\hline
\end{tabular}




\section{Paleoambiente Deposicional y Tipo de Materia Orgánica}

La evaluación del ambiente de depósito de las facies orgánicas que generaron los crudos analizados se estableció con base en el análisis de los esteranos y la relación pristano/fitano extraídos de los fragmentogramas m/z 217 (FIGURA 9) y m/z 99 (FIGURA 7). La cantidad porcentual de los esteranos $\mathrm{C}_{27}$ a $\mathrm{C}_{29}$ muestra que los crudos analizados entran dentro del rango mixto/parálico para la procedencia de la materia orgánica precursora (FIGURA 11). Estos datos se confirman con la relación pristano/fitano cuyo valor superior a uno apunta hacia un medio de depósito subóxico. Por otro lado, las bajas concentraciones de azufre en el intervalo comprendido entre 0,1-0,11 $\%$ (promedio de $0,048 \%$ ) apoyan la tesis de una roca clástica como la roca madre de los crudos analizados. Este tipo de rocas son características de ambientes transicionales subóxicos apuntado por los parámetros anteriores.

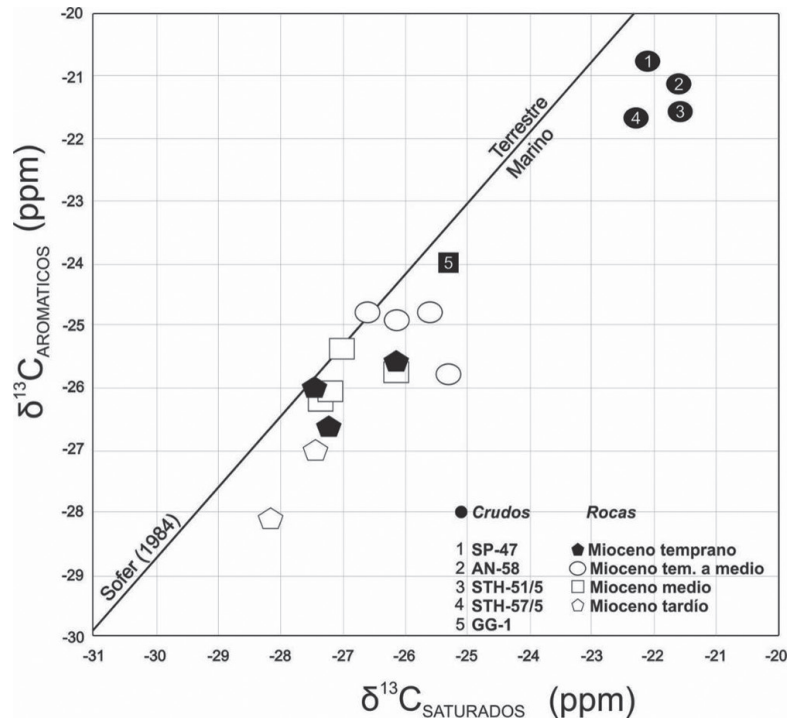

FIGURA 10. Gráfico de Sofer (1984) aplicado sobre los extractos orgánicos de las muestras estudiadas.

Caracterización de rocas: Las muestras de rocas fueron caracterizadas con base en el Porcentaje de Carbono Total, los datos de Rock-Eval $\left(\mathrm{S}_{2} \mathrm{y} \mathrm{T}_{\text {max }}\right.$ ) y reflectancia de la vitrinita por intervalo de edad conocida (TABLA 3). Se analizó el intervalo correspondiente al Mioceno Temprano (Formación Dos Bocas?) del pozo STD-1, generando valores de COT $\%$ de $0,95 \%$ (promedio de cuatro muestras) y un $\mathrm{S}_{2}$ de $0,58 \mathrm{mg} / \mathrm{g}$ de roca lo que implica que tienen un pobre potencial generador. Por otro lado, las rocas del intervalo presentado infieren inmadurez dado los valores de $\mathrm{T}_{\max }$ entre $423^{\circ}-429^{\circ}$ y una reflectancia de la vitrinita entorno a 0,47 . Para el mismo intervalo temporal en rocas analizadas en los pozos D1, GG-1 y AS-1, se obtuvieron los siguientes datos promedios, COT $\% 1,07 \%, \mathrm{~S}_{2}, 0,66 \mathrm{mg} / \mathrm{g}$ de roca seca (promedio de 6 muestras), lo que concuerda en que se trata de una roca con bajo potencial generador. Igualmente, los valores de $\mathrm{T}_{\text {max }}$ de $428^{\circ}$ y la reflectancia de la vitrinita con $0,40 \% \mathrm{R}_{\mathrm{o}}$ infieren inmadurez.

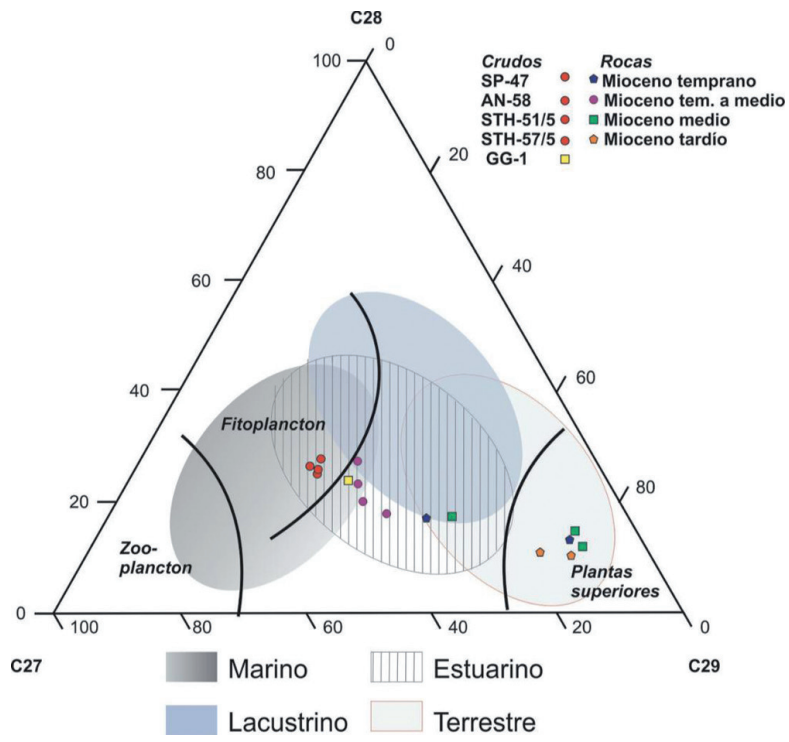

FIGURA 11. Diagrama triangular mostrando la contribución de los esteranos regulares $\alpha \alpha \alpha$ y $\alpha \beta \beta \quad \mathrm{C}_{27}, \mathrm{C}_{28}$ y $\mathrm{C}_{29}$. La interpretación paleoambiental y de fuente acorde a Huang y Meinschein (1979).

Otro intervalo analizado en el pozo STD-1 es el correspondiente al Mioceno medio (Formación Subibaja?). Este intervalo presenta un COT\% promedio de $1,1 \%$ en peso (promedio de 5 muestras) $\mathrm{y}$ un $\mathrm{S}_{2}$ de $3,15 \mathrm{mg} / \mathrm{gr}$ de roca seca, sugiriendo que este intervalo rocoso posee un potencial marginal para generar hidrocarburos. Sin embargo, los datos de madurez termal apuntan a que las rocas son inmaduras dado que la $\mathrm{T}_{\text {max }}$ presenta valores entre $401-432{ }^{\circ} \mathrm{C}$ y una reflectancia de la vitrinita de 0,42 a $0,53 \% R_{0}$.

TABLA 3. Parámetros de caracterización geoquímica de las rocas (valores promediados).

\begin{tabular}{llcccc}
\hline Edad Geológica & Pozos & \%COT & $\begin{array}{c}\mathbf{S}_{\mathbf{2}} \\
(\mathbf{m g} / \mathbf{g})\end{array}$ & $\mathbf{T}_{\max }$ & $\mathbf{\%} \mathbf{R}_{\mathbf{0}}$ \\
\hline \multirow{2}{*}{$\begin{array}{l}\text { Mioceno } \\
\text { Temprano }\end{array}$} & STD-1 & 0,96 & 0,58 & 426 & 0,49 \\
& AS-1, & 1,04 & 1,62 & 425 & 0,41 \\
\multirow{2}{*}{ Mioceno Medio } & STD-1 & 1,1 & 3,15 & 417 & 0,46 \\
& D1 & 2,04 & 5,28 & 432 & 0,44 \\
Mioceno Tardío & D1 & 1,92 & 4,39 & 433 & 0,50 \\
\hline
\end{tabular}


El intervalo correspondiente al Mioceno medio también fue analizado en los pozos D-1, GG-1 y AS-1 en el Golfo de Guayaquil. Los valores obtenidos para COT \% estuvieron en torno a $1,04 \%$ de promedio y unos valores de $\mathrm{S}_{2}$ superiores a $1,00 \mathrm{mg} / \mathrm{g}$ de roca seca. Esto infiere que el intervalo analizado tiene potencial generador de hidrocarburos tal y como se había apuntado en los datos obtenidos del pozo STD1 , sin embargo, de manera análoga al citado pozo los valores de madurez termal en el caso que nos ocupa señala hacia condiciones de inmadurez en la roca $\left(\mathrm{T}_{\max }\right.$ es $425^{\circ} \mathrm{C}$ y una $\% R_{o}$ de $0,41 \%$ ).

El intervalo rocoso de edad Mioceno tardío (Formación Progreso) extraído del STD-1 presentó unos valores de COT de $0,70 \%$ y un $\mathrm{S}_{2}$ de $1,62 \mathrm{mg} / \mathrm{g}$ de roca seca, lo que indica un bajo potencial generador de hidrocarburos. El mismo intervalo rocoso en el pozo D-1 arroja valores de COT entorno al 2,04\% y un valor promedio de 5,28 $\mathrm{mg} / \mathrm{g}$ de roca seca (promedio de cinco muestras), lo que confirma el bajo potencial generador obtenido en el pozo STD-1. Sin embargo las muestras de rocas analizadas del pozo D-1 dieron como resultado valores de COT\% de $1,92 \%$ y $4,39 \mathrm{~S}_{2}$ que infieren un cierto potencial generador de hidrocarburos, y pese a presentar inmadurez la $\mathrm{T}_{\max }$ se muestra próxima la ventana de petróleo y una reflectancia de la vitrinita del $0,5 \% R_{\mathrm{o}}$.

Materia orgánica parental en las rocas: Los resultados obtenidos a partir del análisis Rock-Eval del cálculo del índice de hidrogeno (IH), índice de oxigeno (IO) fueron representados en el Diagrama de van Krevelen modificado (FIGURA 12). Como se puede observar, las rocas representadas entran dentro del campo de la materia orgánica terrestre.

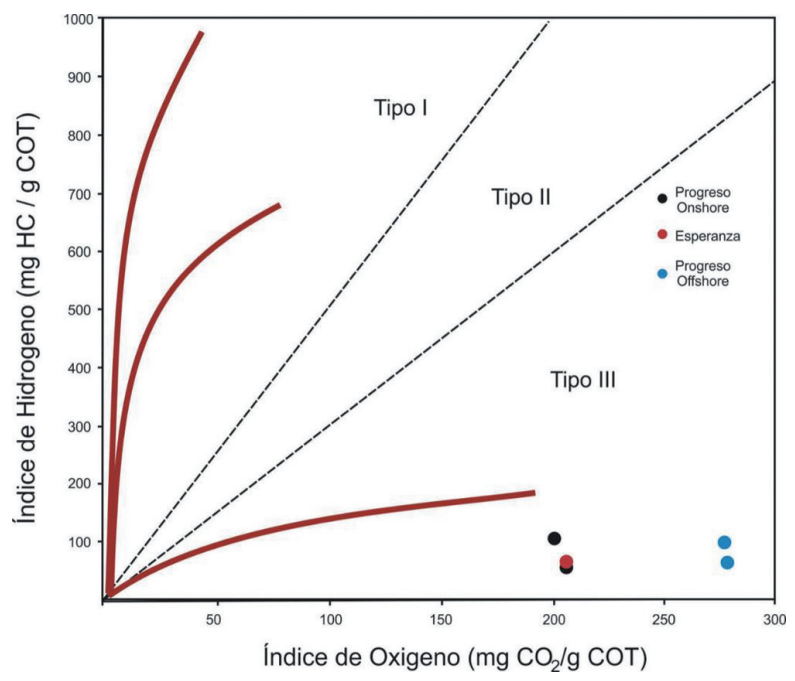

FIGURA 12. Diagrama de van Krevelen a partir de datos de la pirólisis Rock-Eval en las muestras de roca del presente estudio.

\section{Correlación Crudo-Crudo}

Algunas características generales y parámetros geoquímicos determinados en los crudos y sus fracciones constituyentes sugieren, en primera aproximación, que los crudos estudiados son similares entre sí. Así, la composición SARA (FIGURA 4), las relaciones entre isoprenoides (FIGURA 5) al igual que la distribución de esteranos y terpanos pentacíclicos (FIGURAS 8 y 9) es similar. Por último, la interpretación de los ambientes sedimentarios para la posible roca madre no muestra mayores diferencias; sin embargo, la secuencia sedimentaria de la Cuenca Progreso no presenta diferencias significativas a lo largo de la columna; de hecho, predominan y se repiten las secuencias propias de medios turbidíticos no muy lejanos a aportes estuarinos.

Los efectos de la biodegradación detectada -nivel 3-4 según la escala de biodegradación de Peters et al. (2005)- pueden estar enmascarando los parámetros geoquímicos en los crudos afectados, lo que complica una eventual correlación. Por el contrario, la huella isotópica no es afectada por biodegradación, en las fracciones $\mathrm{C}_{15^{+}}$(Peters et al., 2005) por lo que en el diagrama de Sofer (1984) correspondiente a la FIGURA 10 , la distribución isotópica distintiva de la muestra del Golfo de Guayaquil GG-1 respecto a los restantes crudos no puede ser atribuida a biodegradación (Vieth y Wilkes, 2010). La diferencia de más de un 2-3\%o en la distribución isotópica (Peters et al., 2005) para este crudo respecto a los restantes permite proponer que al menos este último no es correlacionable con los restantes. Así, a pesar de las similitudes geoquímicas, en el Golfo de Guayaquil hay al menos dos familias genéticas diferentes.

\section{Correlación Crudo-Roca}

Los crudos analizados se dividen en dos familias genéticas, hecho fundamentado en el análisis isotópico como muestra el diagrama de Sofer (FIGURA 10), pese a que la materia orgánica precursora de los mismos parece tener el mismo origen en un medio marinotransicional. La primera familia, correspondiente a los crudos de la Península de Santa Elena (onshore), ha sido relacionada tradicionalmente con las formaciones Dos Bocas-Villingota, de origen marino. No obstante, éstas no son correlacionables in situ, dada la inmadurez de éstas. Por otro lado, la segunda familia correspondiente al crudo de Golfo de Guayaquil ha sido correlacionada con la Formación del Mioceno medio, Subibaja, de origen marino y con madurez incipiente. 


\section{CONCLUSIONES}

Se reconocen dos tipos genéticos de crudo, los crudos onshore de la Península y el crudo del Golfo de Guayaquil.

Las distribuciones de biomarcadores no permiten discriminar entre los crudos, en parte debido a la similitud de la calidad de la materia orgánica precursora, y del nivel alcanzado por los crudos. Sin embargo, la huella isotópica es notablemente diferente en el crudo offshore del Golfo de Guayaquil respecto a los restantes, correspondientes al área de Ancón y la Península, por lo que queda evidenciada la presencia de dos tipos de crudo, muy similares entre sí, pero con una importante diferencia a nivel isotópico. Por tanto, se propone la existencia de dos sistemas petroleros diferentes en el área de estudio.

La materia orgánica parental de los crudos analizados es de origen marino, con significativo aporte terrígeno depositado en ambiente subóxico. Posiblemente se trata de un ambiente de transición estuarino.

Los crudos analizados presentan madurez térmica característica de inicio de ventana de petróleo.

Las rocas analizadas son térmicamente inmaduras por lo que no han podido generar los crudos analizados.

La roca madre del crudo GG-1 se presenta como una roca clástica de origen marino o transicional, con aporte de materia orgánica continental, posiblemente de tipo estuarino, que respondería a las condiciones subóxicas. En concordancia con ello la posible roca madre puede estar conformada por los depósitos del intervalo Mioceno medio (Pozo D-1), la cual puede ser la Formación Subibaja o su equivalente lateral en Golfo de Guayaquil.

La roca generadora de los crudos de la Península de Santa Elena, se postula como una roca madre marina/ transicional. Con esas condiciones los candidatos disponibles corresponden a depósitos del Mioceno temprano, Dos Bocas/Villingota; sin embargo, las muestras analizadas de la misma presentan inmadurez. Cabe señalar la posibilidad de que esta formación haya alcanzado niveles de madurez mayor hacia el centro del Golfo y por migración los yacimientos se localizan onshore. Otra posibilidad que no se puede descartar es que la roca madre provenga de otros intervalos de la columna estratigráfica ausentes en los pozos analizados, o inclusive en el Cretácico.
No se puede descartar la existencia de una roca madre no identificada hasta el momento que haya generado los crudos de la Península de Santa Elena.

\section{AGRADECIMIENTOS}

A la Universidad Estatal Península de Santa Elena. A través del proyecto "Geoquímica de los Crudos del Litoral ecuatoriano (CUP: 91870000.0000 .382443$)$ ".

\section{REFERENCIAS}

ASTM D3279-07. Standard Test Method for n-Hepptane Insolubles. American National Standard, Vol. 64. 2008.

Benítez, S. (1995). Évolution géodynamique de la province côtiere sud-équatorienne au Crétacé supérieur-Tertiaire. Geologie Alpine, 71, 3-163.

Cobos, L. (2010). Estudio integrado del Golfo de Guayaquil del Mioceno al Reciente. Tesis de Grado. Facultad de Ingeniería en Ciencias de la Tierra. Escuela Superior Politécnica del Litoral. Ecuador.

Deniaud, Y. (1998). Evolución tectono-sedimentaria de las cuencas costeras Neógenas del Ecuador. Convenio Petroproduccion / ORSTOM. Informe Interno. Quito, Ecuador.

Deniaud, Y., Baby, P., Basile, C., Ordoñez, M., Montenegro, G., and Mascle, G. (1999). Ouverture et évolution tectono-sedimentaire du golfe de Guayaquil: Bassin d'avant-arc néogène et quaternaire du Sud des Andes équatoriennes. Comptes Rendus de l'Académie des Sciences Series IIA - Earth and Planetary Science, 328(3), 181-187. doi: 10.1016/S1251-8050(99)80094-9.

Escobar-Navarro, M. (2008). Fundamentos de la Geoquímica del Petróleo. XI Congreso Latinoamericano de Geoquímica Orgánica, Isla de Margarita, Venezuela.

Huang, W., and Meinschein, W. (1979). Sterols as ecological indicators. Geochimica et Cosmochimica Acta, 43(5), 739-745. doi: 10.1016/0016-7037(79)90257-6.

Hunt, J.M. (1996). Petroleum geochemistry and geology. (2nd Edition). San Francisco: Freeman and Company. 
Jaillard, E., Lapierre, H., Ordóñez, M., Toro, J., Amórtegui, A., and Vanmelle, J. (2009). Accreted oceanic terranes in Ecuador: southern edge of the Caribbean Plate?. Geological Society, London, Special Publications, 328, 469-485. doi: 10.1144/ SP328.19.

Jaillard, E., Sempere, T., Soler, P., Carlier, G., and Marocco, R. (1995). The role of Tethys in the evolution of the northern Andes between Late Permian and Late Eocene times. In A.E.M. Nairn, L.E. Ricou, B. Vrielynck, J. Dercourt (Eds.), The Tethys Ocean (pp. 463-492). Boston: Springer.

Lijmbach, G. (1975). On the origin of petroleum. 9th World Petroleum Congress. Tokyo, Japan.

Marksteiner, R., and Alemán, A. (1991). Coastal Ecuador technical evaluation agreement. Peroecuador, Guayaquil. Informe Interno. AMOCO.

Moustafa Y. (2004). Environmental assessment of petroleum contamination of Gamasa-Damiette Beaches. Oriental Journal of Chemistry, 20(2), 219-226.

Moustafa, Y., and Morsi, R. (2012). Biomarkers. In S. Dhanarasu (Ed.), Chromatography and its applications (pp. 165-186). Croatia, Rietja: InTech.

Peters, K.E., Walters, C.C., and Moldowan, J.M. (2005). The Biomarker Guide. Vol. 2. New York: Cambridge University Press.

Sofer, Z. (1984). Stable carbon isotope compositions of crude oils: Application to source depositional environments and petroleum alteration. $A A P G$ Bulletin, 68(1), 31-49.

Wallrabe-Adams, H. (1990). Petrology and geotectonic development of the Western Ecuadorian Andes: the Basic Igneous Complex. Tectonophysics, 185(1-2), 163-182. doi: 10.1016/0040-1951(90)90411-Z.

Vieth, A., and Wilkes, H. (2010). Stable isotopes in understanding origin and degradation processes of petroleum. In K. N. Timmis (Ed.), Handbook of hydrocarbon and lipid microbiology (pp. 97111). Berlin: Springer.

\begin{tabular}{cc}
\hline \hline & Erica Lorenzo \\
$\mathbf{4 2}$ & ORCID: 0000-0002-6859-8796
\end{tabular}

Wladimir Andrés Roca-Beltrán

ORCID: 0000-0002-0509-4160

Manuel Martínez

ORCID: 0000-0003-0985-5673

Antonio Morato

ORCID: 0000-0003-2163-5323

Paulo César Escandón-Panchana

ORCID: 0000-0002-7788-3118

Cristian Paul Álvarez-Domínguez

ORCID: 0000-0002-2529-8398

Trabajo recibido: junio 09 de 2017

Trabajo aceptado: octubre 25 de 2017 